\title{
The MASCOT landing area on asteroid (162173) Ryugu: Stereo-photogrammetric analysis using images of the ONC onboard the Hayabusa2 spacecraft
}

\author{
F. Preusker ${ }^{1}$, F. Scholten ${ }^{1}$, S. Elgner ${ }^{1}$, K.-D. Matz ${ }^{1}$, S. Kameda ${ }^{2}$, T. Roatsch ${ }^{1}$, R. Jaumann ${ }^{1}$, S. Sugita ${ }^{3,4}$, R. Honda ${ }^{5}$,
}

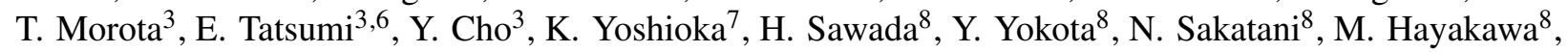
M. Matsuoka ${ }^{8}$, M. Yamada ${ }^{4}$, T. Kouyama ${ }^{9}$, H. Suzuki ${ }^{10}$, C. Honda ${ }^{11}$, and K. Ogawa ${ }^{12}$

${ }^{1}$ German Aerospace Center (DLR), Institute of Planetary Research, 12489 Berlin, Germany e-mail: Frank.Preusker@dlr.de

2 Department of Physics, Rikkyo University, 3-34-1 Nishi-Ikebukuro, Toshima, Tokyo, Japan

3 University of Tokyo, 7-3-1 Hongo, Bunkyo, Tokyo, Japan

${ }^{4}$ Planetary Exploration Research Center, Chiba Institute of Technology, 2-17-1 Tsudanuma, Narashino, Chiba, Japan

5 Department of Information Science, Kochi University, 2-5-1 Akebono, Kochi, Japan

${ }^{6}$ Instituto de Astrofisica de Canarias, University of La Laguna, Santa Cruz de Tenerife, Spain

7 Department of Complexity Science and Engineering, University of Tokyo, 5-1-5 Kashiwanoha, Kashiwa, Chiba, Japan

8 ISAS, JAXA, 3-3-1 Yoshinodai, Chuo, Sagamihara, Kanagawa, Japan

9 National Institute of Advanced Industrial Science and Technology, 2-3-26 Aomi, Koto, Tokyo, Japan

${ }_{10}$ Department of Physics, Meiji University, 1-1-1 Higashimita, Tama, Kawasaki, Kanagawa, Japan

11 CAIST/ARC-Space, University of Aizu, Aizu-Wakamatsu, Fukushima, Japan

12 Department of Planetology, Kobe University, 1-1 Rokkodaicho, Nada, Kobe, Hyogo, Japan

Received 23 September 2019 / Accepted 3 November 2019

\begin{abstract}
A high-resolution 3D surface model, map-projected to a digital terrain model (DTM), and precisely ortho-rectified context images (orthoimages) of MASCOT landing site area are important data sets for the scientific analysis of relevant data that have been acquired with MASCOT's image camera system MASCam and other instruments (e.g., the radiometer MARA and the magnetometer MASMag). We performed a stereo-photogrammetric (SPG) analysis of 1050 images acquired from the Hayabusa2 Optical Navigation Camera system (ONC) during the asteroid characterization phase and the MASCOT release phase in early October 2018 to construct a photogrammetric control point network of asteroid (162173) Ryugu. We validated existing rotational parameters for Ryugu and improved the camera orientation (position and pointing) of the ONC images to decimeter accuracy using SPG bundle block adjustment. We produced a high-resolution DTM of the entire MASCOT landing site area. Finally, based on this DTM, a set of orthoimages from the highest-resolution ONC images around MASCOT's final rest position complements the results of this analysis.
\end{abstract}

Key words. minor planets, asteroids: general - planets and satellites: surfaces - minor planets, asteroids: individual: (162173) Ryugu

\section{Introduction}

In December 2014, the Japan Aerospace Exploration Agency (JAXA) launched its Hayabusa2 spacecraft to explore and return samples of the C-type near-earth asteroid (162173) Ryugu (Watanabe et al. 2017). On 27 June 2019, the Hayabusa2 (HY2) spacecraft reached its Home Position near Ryugu (Watanabe et al. 2019). At this Home Position from an altitude of about $20 \mathrm{~km}$ above Ryugu, the surface of the asteroid was continuously imaged with the Optical Navigation Camera system (ONC, Kameda et al. 2017). Additionally, laser tracks were acquired using the onboard light and ranging detection system (LIDAR, Mizuno et al. 2017). Within an asteroid characterization phase (July-September 2018), the ONC acquired about 1500 images of the illuminated surface of Ryugu using the narrow angle camera ONC-T (Sugita et al. 2019). This image data set allows the creation of a global control point network as well as the determination of the rotational parameters of Ryugu with the use of stereo-photogrammetric (SPG) methods. Among other instruments, the HY2 spacecraft is equipped with the lander module Mobile Asteroid Surface Scout (MASCOT, Ho et al. 2017). On 2 October 2018, the HY2 spacecraft left its Home Position with the approach maneuver to the surface of Ryugu for the release of MASCOT on 3 October 2018 at 1:57:19 UTC (Jaumann et al. 2019; Scholten et al. 2019a). The ONC acquired continuously images of the landing site and the surrounding area before, during, and after MASCOT's release. On 4 October 2018, the MASCOT release operation ended with the return of HY2 to its Home Position. In this article we describe the construction of a photogrammetric control network to improve the accuracy of the camera orientation of the images from the asteroid characterization phase as well as the verification of the body-fixed frame of Ryugu (see Sect. 2). Based on these results, we extended the control point network using images from the MASCOT release phase and derived a high-resolution surface model (see Sect. 3.2) and ortho-rectified context images (orthoimages) from MASCOT's landing site area (see Sect. 3.3). 


\section{Global context for MASCOT's landing area}

The main task for the high-precision registration and representation of all MASCOT relevant observations is to construct a control point network. Here, a control point network is a global dataset, which consists of a list of surface points associated with a list of respective images and image coordinates. These surface points represent surface features, for which body-fixed coordinates are precisely known. The realization of such a control point network is an essential part of SPG processing, which is based on algorithms and methods developed at German Aerospace Center (DLR) in the last 30 years and applied successfully in different planetary missions (Preusker et al. 2017a,b; Scholten et al. 2012; Gwinner et al. 2009, 2010). Here, the three major steps are the selection of sets of stereo pairs (stereo models as a combination of at least three images) within the observation phases (Sect. 2.2), the measurement of tie-points within the selected stereo pairs by means of stereo image matching (Sect. 2.3), and the SPG bundle block adjustment in order to determine improved orientation of the images as well as the high-precision surface points (Sect. 2.4). In addition, the rotational state of Ryugu can be controlled indirectly by variations of the rotational parameters within SPG bundle block adjustment (Preusker et al. 2015). Finally, these results yield the basic framework for the generation of highly accurate data products for the MASCOT landing site (Sect. 3).

\subsection{Ancillary data}

All data used in SPG processing in addition to the image data are available in the form of SPICE kernels (Acton 1996). They provide (initial) information about the position and orientation of the instruments (e.g., the ONC), of the spacecraft, and of other relevant bodies (e.g., Ryugu and the Sun) relative to the inertial Equatorial J2000 coordinate frame (SPK and CK kernels). Additional frames defined for the spacecraft, its subsystems, and instruments are provided in the frame kernel (FK). The geometric information of the instruments (including camera distortion) is stored in the instrument kernel (IK). Finally, the planetary constant kernel (PCK) describes the transformation between the J2000 frame and the body-fixed frame (BFF). We used the mission kernels provided for the science team for all the processing steps described in this article. However, we have included the information about the eccentricity of the entrance pupils of the ONC relative to the HY2 spacecraft frame (Suzuki et al. 2018) into an additional kernel.

\subsection{Stereo-image selection and derived stereo pairs}

The ONC onboard HY2 consisted of three $1024 \times 1024$ pixel CCD framing cameras: the narrow-angle camera ONC-T is equipped with a filter wheel with seven filters for color observations (Kameda et al. 2017; Suzuki et al. 2018; Tatsumi et al. 2019) and the wide-angle cameras ONC-W1 and ONC-W2 are designed for monochrome observations only. Due to the small focal length and a 12 times larger Instantaneous Field of View (IFOV) of the wide-angle cameras compared to ONC-T, they only become relevant for the reconstruction of the MASCOT landing site area (Sect. 3). Within the asteroid characterization phase, ONC acquired about 1500 ONC-T images (Sugita et al. 2019), most of them from HY2's Home Position at about $20 \mathrm{~km}$ attitude and at 3-7 km attitude (Box-A and Box-B; Box-C and Mid-Alt; see Watanabe et al. 2019, Fig. S1). For the development of a global network, only those observation periods were selected, which comprise complete $360^{\circ}$ coverage (equivalent to a rotational movie) or provide additional information in terms of
Table 1. Selection of ONC images for the construction of the global control network for (162173) Ryugu and for the reconstruction of MASCOT landing site area.

\begin{tabular}{lccc}
\hline \hline $\begin{array}{l}\text { Observation } \\
\text { phase }\end{array}$ & $\begin{array}{c}\text { Number of } \\
\text { images }\end{array}$ & $\begin{array}{c}\text { Average } \\
\text { pixel scale } \\
\left.\text { [m pixel }{ }^{-1}\right]\end{array}$ & $\begin{array}{c}\text { HY2 } \\
\text { operation/phase }\end{array}$ \\
\hline $2018-06-30$ & 127 & 2.2 & BOX-A \\
$2018-07-03$ & 91 & 2.2 & BOX-A \\
$2018-07-10$ & 127 & 2.1 & BOX-A \\
$2018-07-12$ & 84 & 2.1 & BOX-A \\
$2018-07-19$ & 28 & $1.10-1.30$ & BOX-C \\
$2018-07-20$ & 101 & $0.64-0.68$ & MID-ALT \\
$2018-08-01$ & 179 & $0.55-0.76$ & MID-ALT \\
$2018-08-06$ & 58 & $0.10-0.44$ & Gravity \\
$2018-08-24$ & 129 & 2.6 & BOX-B \\
$2018-09-11$ & 14 & $0.31-0.97$ & Touchdown test \\
$2018-09-21$ & 10 & $0.02-0.10$ & MINERVA II release \\
\hline $2018-10-02$ & 2 & $0.20-0.75$ & MID-ALT \\
$2018-10-03$ & 75 & $0.02-0.50$ & MASCOT release \\
$2018-10-04$ & 25 & $0.29-0.31$ & MID-ALT \\
\hline
\end{tabular}

higher image resolution. In total, we selected 948 ONC-T images (see Table 1 top rows). Due to the constant spatial constellation between the Sun, HY2 spacecraft, and Ryugu, illumination conditions during the different observation phases are almost identical. Therefore, the selection and definition of stereo-image pairs was only based on image overlap, stereo angle, and image scale and not limited by variable illumination conditions. Finally, we defined a total of 13370 stereo pairs with a minimum of three and up to eight images.

\subsection{Stereo image matching}

For each of the 13370 defined stereo models, we use SPG area-based image-matching technique (Wewel 1996) to derive image coordinates of identical points within the images. Here, we applied the image matching in a more sparse way (about every thirtieth pixel) to generate a reduced set of tie-points that serves as input for the subsequent SPG bundle block adjustment. However, for the derivation of points for the final 3D surface model (Sect. 3.2), this grid was as dense as the original pixel size of the respective images. The derived set of tie-points for the bundle block adjustment comprises about 38000 tie-points with about 160000 image point measurements.

\subsection{Stereo-photogrammetric bundle block adjustment of ONC positions and attitude}

The central step within SPG is the bundle block adjustment (Zhang et al. 1996). This comprises a least-squares adjustment of observations, typically image coordinates of homologous points (tie-points) within (multi-)stereo images, in order to determine the unknown parameters, namely six camera orientation parameters (three metric parameters for the camera position and three angular parameters for the camera pointing) and three coordinates for the tie-point coordinates in object space. For redundancy and stability, each tie-point must be defined in a set of at least three stereo images. The relation between tie-point coordinates and the corresponding surface point is mathematically defined through so-called collinearity equations (Albertz \& Wiggenhagen 2009). In addition, LIDAR's range observations were introduced as conditional equations in 
Table 2. Spin pole and spin rate solutions of Ryugu.

\begin{tabular}{|c|c|c|c|}
\hline Reference & $\begin{array}{c}\text { Right ascension } \\
{\left[{ }^{\circ}\right]}\end{array}$ & $\begin{array}{c}\text { Declination } \\
{\left[{ }^{\circ}\right]}\end{array}$ & $\begin{array}{c}\text { Spin rate } \\
\text { [degree per day] }\end{array}$ \\
\hline $\begin{array}{l}\text { PCK v20180829 } \\
\text { (Watanabe et al. 2019) }\end{array}$ & 96.3979 & -66.3987 & 1131.98308 \\
\hline $\begin{array}{l}\text { PCK v20181014 } \\
\text { (Hirata 2018) }\end{array}$ & 96.4010 & -66.3995 & 1131.98324 \\
\hline This investigation & 96.3956 & -66.3937 & 1131.98280 \\
\hline
\end{tabular}

order to constrain the absolute scale. The results of the bundle block adjustment are improved camera positions and attitudes for the entire set of 948 involved ONC images with accuracies at the sub-pixel level.

\subsection{Verification of the rotational state of Ryugu}

The SPG bundle block adjustment is performed in the bodyfixed coordinate system and corrects for random errors within initial orientation. Therefore, systematic variations from any uncertainty of the rotational elements, which describe the transformation from the inertial coordinate frame J2000 to the bodyfixed coordinate frame, cannot be determined directly. However, the determination of the rotational elements can be carried out indirectly by minimizing the stress within the adjusted image block by means of brute-force-like variations of the rotational elements. The subsequent analysis of the series of iterative bundle block adjustment results (e.g., of the achieved a-posteriori accuracies) can be used to estimate the spin rate as well as the spin pole of the target body (see Preusker et al. 2015). We determined the pole right ascension $\alpha_{0}=\left(96.3956 \pm 0.0082^{\circ}\right)$, pole declination $\delta_{0}=\left(-66.3937 \pm 0.0063^{\circ}\right)$, and spin rate $W_{1}=$ $(1131.98280 \pm 0.00055 \mathrm{deg} /$ day $)$. Our results show very good agreement with other solutions of rotational states within the HY2 project (see Table 2). Effects of the deviations to these solutions do not exceed $20 \mathrm{~cm}$ on Ryugu's surface. The impact on the results of the bundle block adjustment is negligible. Therefore, for consistency and comparability with other investigations, we adopted for our analysis the PCK v20181014 (previously selected as the common definition of Ryugu's body-fixed frame for any MASCOT-related science) in SPICE PCK format:

BODY2162173_POLE_RA $=(96.4010$ 0. 0.)

BODY2162173_POLE_Dec $=(-66.3995$ 0. 0.)

BODY2162173_PM $=(271.23941131 .98324$ 0. $)$

\section{MASCOT landing site area}

The best pixel scale of the images from the asteroid characterization phase showing MASCOT's landing site area is $55 \mathrm{~cm} \mathrm{pixel}^{-1}$ (see Table 1 bottom rows). About 120 ONC images were acquired during MASCOT's release operation phase from 2 to 4 October. From these, we selected 102 images (70 ONC-T, 23 ONC-W1, and 9 ONC-W2 images) that have pixel scales from 5 to $50 \mathrm{~cm} \mathrm{pixel}^{-1}$ and used them for the following reconstruction of the trajectory of HY2 during MASCOT's release phase and for the characterization of the landing site area.

\subsection{Reconstruction of the Hayabusa2 descent and ascent trajectory during MASCOT release operation phase}

With the processing steps described in Sect. 2, we set up an extended control network, which combined the high-resolution
Table 3. Improved ONC positions in Ryugu's body-fixed frame for the MASCOT release phase from SPG bundle block adjustment.

\begin{tabular}{|c|c|c|c|c|c|}
\hline Camera & Image time & Image ID & $X[\mathrm{~m}]$ & $Y[\mathrm{~m}]$ & $Z[\mathrm{~m}]$ \\
\hline \multicolumn{6}{|c|}{ Descent } \\
\hline ONC-W1 & $01: 46: 52$ & 53215 & 412.175 & -257.168 & -211.565 \\
\hline ONC-W1 & 01:49:00 & 53221 & 394.597 & -262.295 & -211.805 \\
\hline ONC-W1 & 01:51:08 & 53227 & 377.357 & -266.894 & -211.306 \\
\hline ONC-W1 & $01: 53: 16$ & 53233 & 360.536 & -271.089 & -210.051 \\
\hline ONC-W2 & $01: 56: 54$ & 53137 & 338.297 & -280.683 & -206.337 \\
\hline ONC-W2 & 01:57:04 & 53140 & 337.343 & -281.228 & -206.089 \\
\hline ONC-W2 & $01: 57: 14$ & 53143 & 336.434 & -281.816 & -205.898 \\
\hline ONC-W2 & $01: 57: 24$ & 53146 & 335.465 & -282.341 & -205.656 \\
\hline ONC-W2 & 01:57:34 & & 334.506 & -282.903 & -205.411 \\
\hline ONC-W2 & 01:57:44 & 53152 & 333.513 & -283.399 & -205.163 \\
\hline ONC-W2 & 01:57:54 & & 332.538 & -283.928 & -204.925 \\
\hline ONC-W2 & 04 & 3158 & 331. & -284.417 & -204.658 \\
\hline & & 53161 & 330.581 & -284.960 & -204.431 \\
\hline \multicolumn{6}{|c|}{ Ascent } \\
\hline ONC-W1 & $01: 59: 40$ & 53164 & 346.238 & -314.469 & -204.774 \\
\hline ONC-W1 & 12 & 53167 & 353 & -326.696 & -204.952 \\
\hline ONC-W1 & 02:00 & 53170 & 360. & -338.926 & -205.095 \\
\hline ONC-W1 & 02:01:16 & 53173 & 367.267 & -351.150 & -205.144 \\
\hline ONC-W1 & 02:01:48 & 53176 & 373.835 & -363.529 & -205.179 \\
\hline ONC-W1 & 02:02:20 & 53179 & 380.205 & -375.932 & -205.184 \\
\hline ONC-W1 & 02:02:52 & & 386. & -388.383 & -205.135 \\
\hline ONC-W1 & 02:03:24 & 53185 & 391.933 & -401 & -205.086 \\
\hline ONC-W1 & 02:0 & 53188 & 398. & -413 & -204.931 \\
\hline ONC-W1 & 02:0 & 531 & 403.5 & -426.095 & -204.689 \\
\hline ONC-W1 & 02:05:00 & & 408.474 & -438.956 & -204.450 \\
\hline ONC-W1 & 02:05:32 & 53197 & 413.793 & -451.491 & -204.232 \\
\hline ONC-W1 & 02:06:04 & 53200 & 418.581 & -464.241 & -204.083 \\
\hline ONC-W1 & 02:06:36 & 53203 & 423.276 & -477.091 & -203.936 \\
\hline ONC-W1 & 02:07:08 & 53206 & 427.626 & -489.998 & -203.505 \\
\hline ONC-W1 & 2:07:40 & 53209 & 431.834 & -502.926 & -203.121 \\
\hline ONC-W1 & 02:08:12 & 53212 & 435.829 & -515.739 & -202.878 \\
\hline ONC-T & 02:08:44 & 53239 & 439.400 & -528.912 & -202.704 \\
\hline ONC-W1 & $02: 11: 24$ & 53242 & 455.482 & -594.240 & -200.810 \\
\hline ONC-T & 02:11:56 & 53245 & 457.951 & -607.452 & -200.338 \\
\hline ONC-W1 & 02:14:04 & 53248 & 466.390 & -660.426 & -198.425 \\
\hline ONC-T & $02: 14: 36$ & 53251 & 467.820 & -673.822 & -197.936 \\
\hline ONC-T & $02: 17: 48$ & 53257 & 473.391 & -754.208 & -194.757 \\
\hline ONC-T & 02:21:00 & 53263 & 472.093 & -834.972 & -191.268 \\
\hline ONC-T & $02: 23: 40$ & 53269 & 465.867 & -902.644 & -188.044 \\
\hline ONC-T & $02: 26: 52$ & 53275 & 452.238 & -983.752 & -184.291 \\
\hline ONC-T & $02: 29: 32$ & 53281 & 435.753 & -1050.966 & -181.043 \\
\hline ONC-T & 02:32:44 & 53287 & 410.099 & -1131.045 & -177.001 \\
\hline
\end{tabular}

Notes. We note that the image time is part of the full image name (see caption of Fig. 3) and represents the image acquisition time in UTC on 3 October 2018.

landing site area images with the global context images from the asteroid characterization phase. Particularly, the wider range of image scales required a careful definition of stereo model combinations in order to guarantee a successful tie-point image matching. In the end, we defined a total of about one thousand additional stereo models, performed a combined bundle block adjustment of the ONC images for landing site area together with the block of context images, and retrieved improved orientation data for the 102 landing site area images. A vertical profile of a subset of 41 of the resulting ONC positions (see Table 3 ) is displayed in Fig. 1. They also represent the precise (one decimeter accuracy) reconstruction of the trajectory of the HY2 spacecraft at the end of the descent and the beginning of the ascent. The orientations of these 102 ONC images are the input for the following derivation of the 3D surface model and orthoimages of the MASCOT landing site area. 


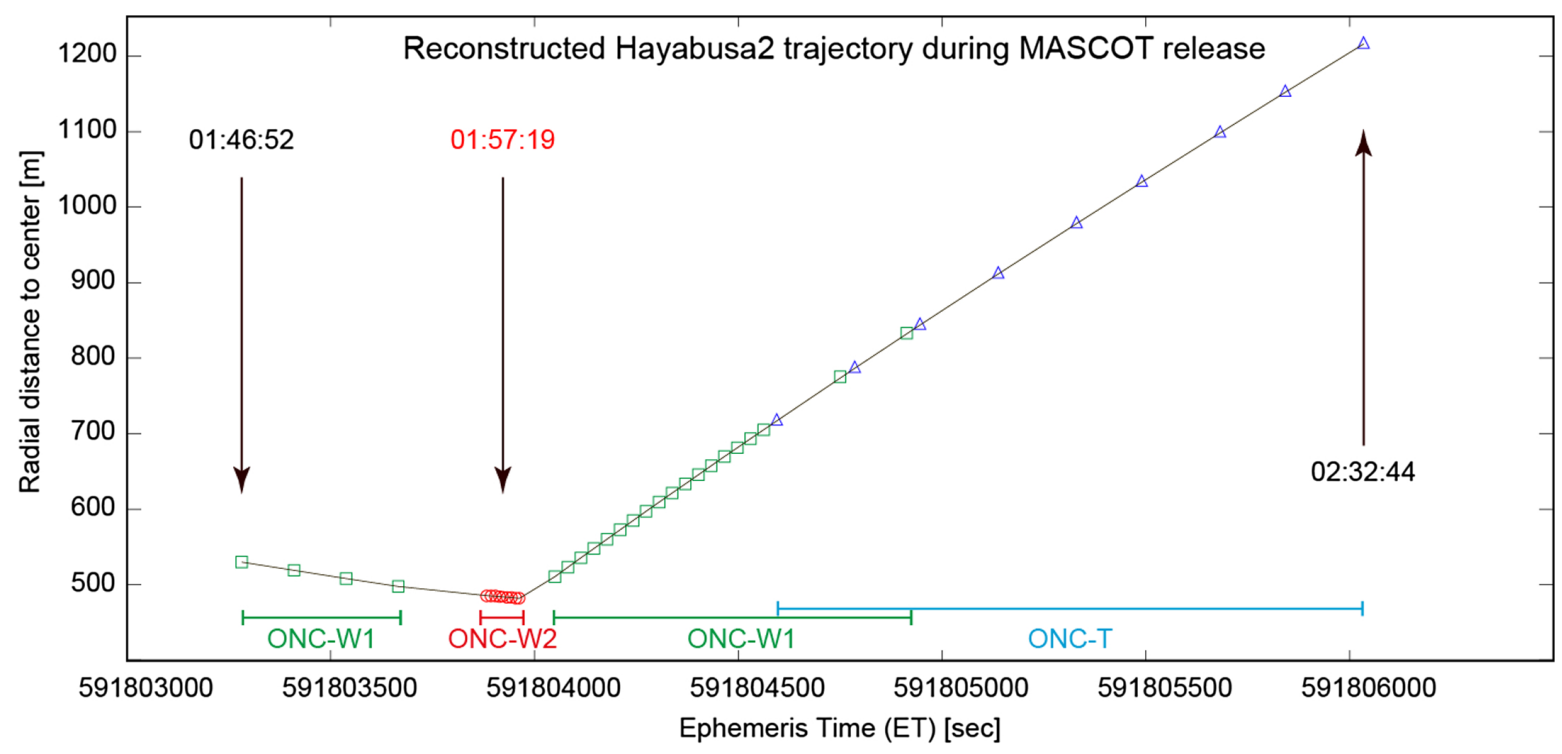

Fig. 1. Vertical profile of the reconstructed descent and ascent trajectory of the Hayabusa2 spacecraft around the release of the MASCOT lander at 01:57:19 UTC. Colored symbols indicate the respective camera positions (green: ONC-W1, red: ONC-W2, blue: ONC-T).
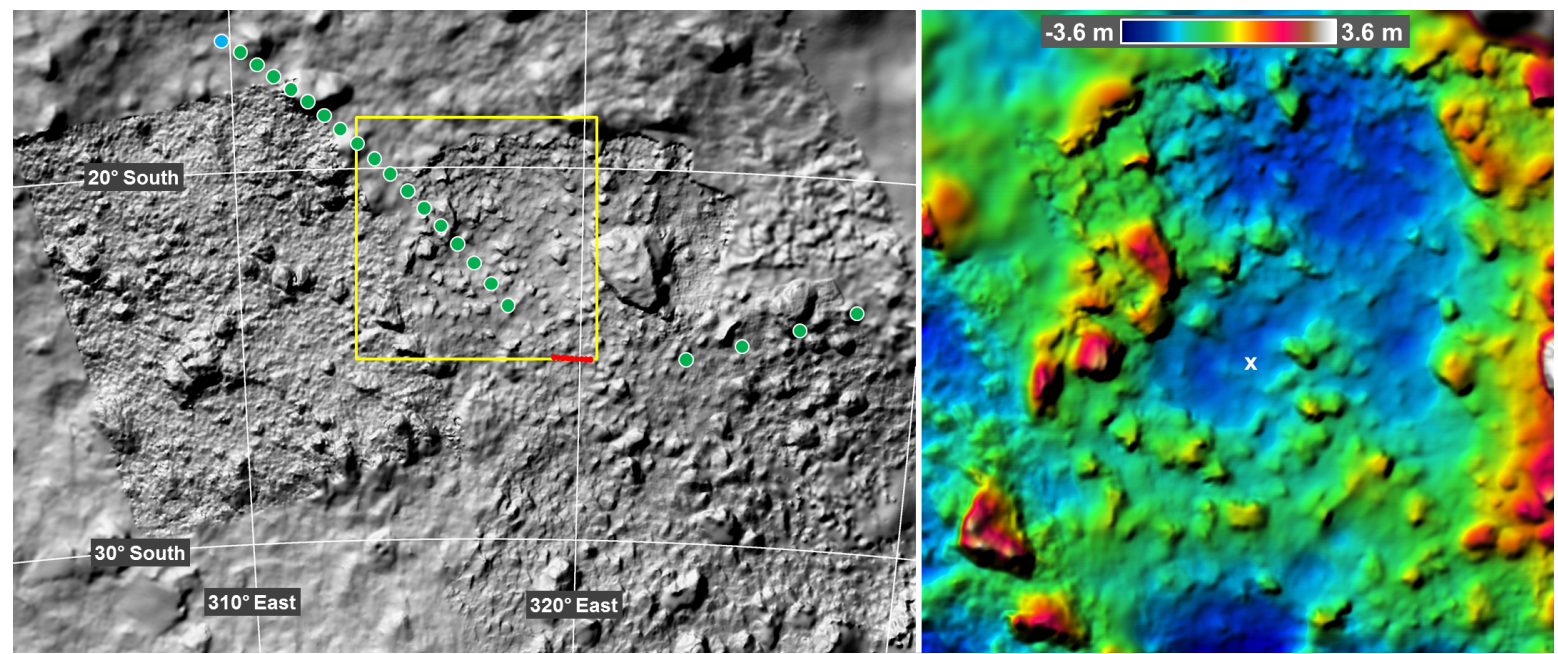

Fig. 2. Three-dimensional surface model of the MASCOT landing site area: left: hill-shaded relief of the regional DTM. The colored dots indicate the sub-spacecraft points at the ONC image acquisition times (green: ONC-W1, red: ONC-W2, blue: ONC-T). Right: color-coded DTM subset of the central $50 \mathrm{~m} \times 50 \mathrm{~m}$ area (represented by the yellow square in the left-hand image) around MASCOT's final rest position (marked by the white cross). For display in this figure, heights were reduced from spherical radii with respect to Ryugu's mean radius to heights above a plane that represents the mean surface slope in this area (about $13^{\circ}$ tilted with respect to the spherical tangent).

\subsection{Generation of a high-resolution 3D surface model of MASCOT landing site area}

Based on the adjusted orientation data for the ONC images of the landing site area and an initial set of 405 billion matched image points, we computed a point cloud of 3D points in Ryugu's body-fixed reference frame by multiple forward ray intersection. Because of the redundancy from at least triple ray intersection, we were able to use the ray intersection accuracy of each $3 \mathrm{D}$ point as a quality criterion using three times the mean intersection error of the entire 3D point cloud as a threshold. The finally remaining 112 billion points provide a mean 3D intersection accuracy of about $10 \mathrm{~cm}$. From these points, we derived a regular grid, which defines the 3D surface model. In Fig. 2, we show this surface model in a $2.5 \mathrm{D}$ representation, known as a digital terrain model (DTM), with a Lambert Azimuthal EqualArea map projection (reference longitude: $317^{\circ}$ east, reference latitude: $22^{\circ}$ south, reference body: sphere with Ryugu's mean radius of $450 \mathrm{~m}$ ). The lateral spacing of the DTM is 128 pixels per degree (about $6 \mathrm{~cm}_{\text {pixel }}{ }^{-1}$ ). The mean accuracy of the final surface model is equivalent to the mean $3 \mathrm{D}$ point accuracy of about $10 \mathrm{~cm}$. The short time span of the high-resolution observation phase (less than one hour) yields no substantial change in illumination and, respectively, no possibility to obtain DTM information in shadowed areas around large boulder-like structures. At these spots, the DTM is based on information from lower resolution observation phases, and therefore its accuracy is degraded (locally up to $1 \mathrm{~m}$ height uncertainty, depending on the size of the respective topographic structure).

\subsection{Precise ortho-rectified ONC context images of MASCOT landing site area}

With the improvement of image orientation data and the generation of a 3D surface model, ONC orthoimages complement the output of this investigation. We chose the same map 


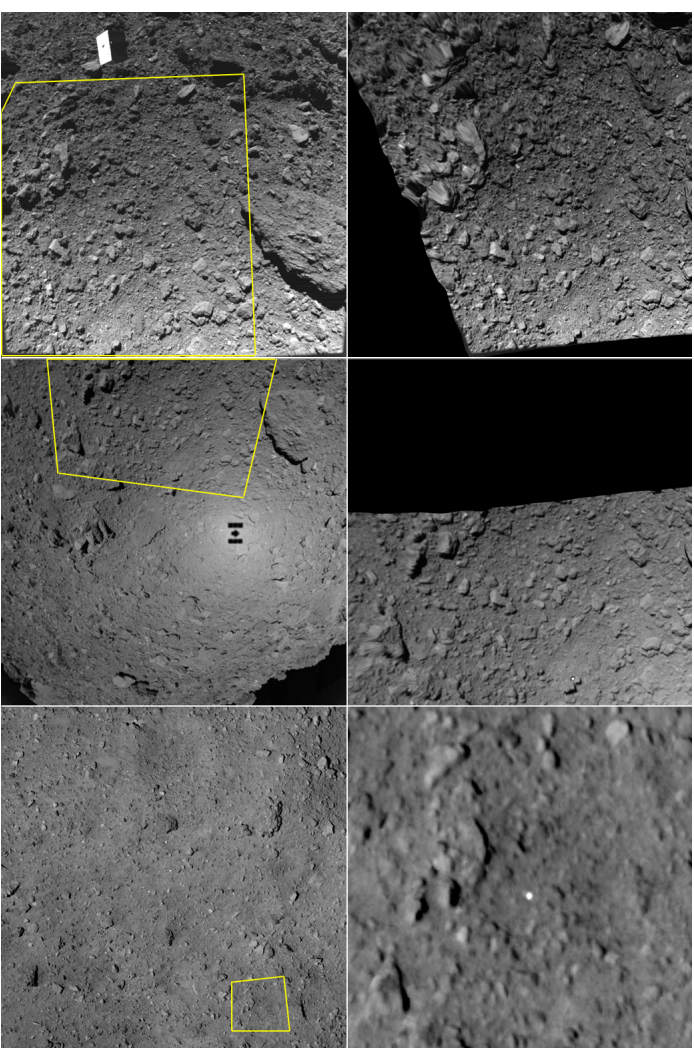

Fig. 3. Typical ONC images covering MASCOT landing site area. Top: ONC-W2 image (hyb2_onc_20181003_015814_w2f_12b), middle: ONC-W1 image (hyb2_onc_20181003_015940_w1f_12b), and bottom: ONC-T image (hyb2_onc_20181004_005509_tvf_12b). Left: original images (stretched for display). Right: orthoimages of the central MASCOT landing site area (see Fig. 2, right). The yellow square indicates the image area of the respective orthoimage.

projection as used for the DTM (see above) and derived orthoimages for those ONC images that cover the central area of $50 \mathrm{~m} \times 50 \mathrm{~m}$ around MASCOT's final rest position on the surface of Ryugu (Scholten et al. 2019a). The accuracy of these orthoimages mainly depends on the vertical accuracy of the DTM. Thus, with a typical vertical accuracy of about $10 \mathrm{~cm}$ for the DTM and with typical emission angles of the ONC images of $<45^{\circ}$, the lateral accuracy of these orthoimages is better than $5 \mathrm{~cm}$. Figure 3 shows typical raw images of the three cameras of the ONC and respective orthoimages. Finally, the high accuracy of our results allows the generation of orthoimages from ONC-T color sequences without color seams. Such color sequences were also acquired during MASCOT's release phase typically from about $3 \mathrm{~km}$ spacecraft altitude (about $30 \mathrm{~cm} \mathrm{pixel}^{-1}$ ). Figure 4 shows an example of a color orthoimage of MASCOT landing site area.

\section{Summary and outlook}

We used SPG methods developed at DLR and derived improved ONC position and pointing information within a combined bundle block adjustment of more than 1000 Hayabusa2 ONC images from a three-month period until MASCOT's release in early October 2018. The achieved 3D accuracy is $0.1 \mathrm{~m}(1 \sigma)$ for the reconstructed descent and ascent trajectory of the HY2 spacecraft as well as for the derived high-resolution ONC data products, namely a 3D surface model and orthoimages of the landing site area of MASCOT. The results and derived data products of this analysis will provide context information for

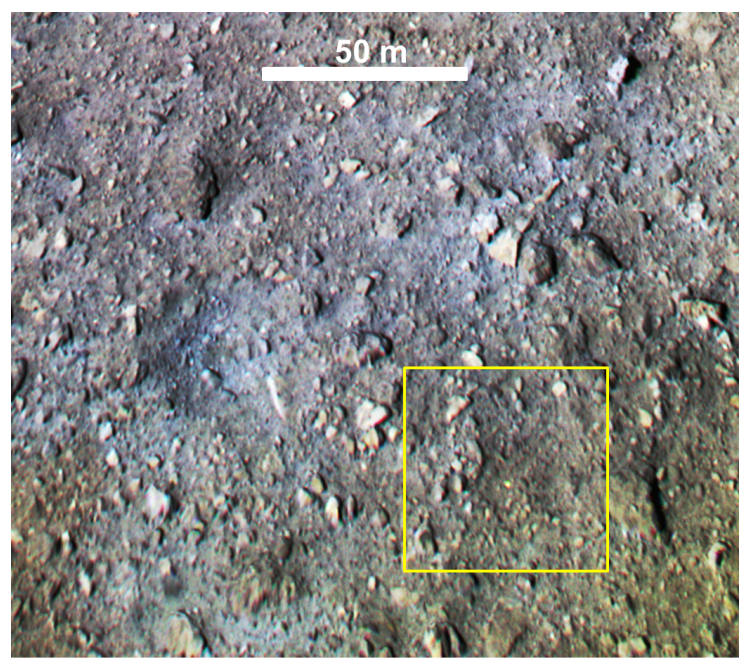

Fig. 4. Example of an ONC-T color orthoimage (stretched for display, no photometric correction or stray light correction applied): hyb2_onc 20181004_005613_tpf_12b $(0.95 \mu \mathrm{m})$ as red, hyb2_onc_20181004_ 005522_twf_12b $(0.70 \mu \mathrm{m})$ as green, and hyb2_onc_20181004_ 005645 tuf_12b $(0.40 \mu \mathrm{m})$ as blue. The yellow square defines the central MASCOT landing site area (see Figs. 2 and 3, right).

subsequent scientific analyses (e.g., about the geology of the MASCOT landing site area) and for the geometric reconstruction of MASCOT's position during its descent and bouncing phase (Scholten et al. 2019a) as well as for its position and orientation during its science cycles on the surface of Ryugu (Scholten et al. $2019 \mathrm{~b})$. The results and data products of this investigation are available at the Europlanet website ${ }^{1}$.

Acknowledgements. The authors acknowledge funding by JAXA, CNES, and DLR, as well as the work of the different instrument teams of Hayabusa2. This work was partially supported by JSPS KAKENHI 17KK0097.

\section{References}

Acton, C. H. 1996, Planet. Space Sci., 44, 65

Albertz, J., \& Wiggenhagen, M. 2009, Guide for Photogrammetry and Remote Sensing (Heidelberg, Germany: Wichmann Verlag)

Gwinner, K., Scholten, F., Spiegel, M., et al. 2009, Photogramm. Eng. Remote Sens., 75, 1127

Gwinner, K., Scholten, F., Preusker, F., et al. 2010, Earth Planet. Sci. Lett., 294, 506

Hirata, N. 2018, PCK Kernel Version 20181014, Pole Information Estimated by Naoyuki Hirata (Kobe Univ.) with SPC. PM/W0 is Adjusted to the Official Definition of Hayabusa2 Project

Ho, T.-M., Baturkin, V., Grimm, C., et al. 2017, Space Sci. Rev., 208, 339 Jaumann, R., Schmitz, N., Ho, T.-M., et al. 2019, Science, 365, 817 Kameda, S., Suzuki, H., Takamatsu, T., et al. 2017, Space Sci. Rev., 208, 17 Mizuno, T., Kase, T., Shiina, T., et al. 2017, Space Sci. Rev., 208, 33 Preusker, F., Scholten, F., Matz, K.-D., et al. 2015, A\&A, 583, A33 Preusker, F., Scholten, F., Matz, K.-D., et al. 2017a, A\&A, 607, L1 Preusker, F., Stark, A., Oberst, J., et al. 2017b, Planet. Space Sci., 142, 26 Scholten, F., Oberst, J., Matz, K. D., et al. 2012, J. Geophys. Res., 117, e00H17 Scholten, F., Preusker, F., Elgner, S., et al. 2019a, A\&A, 632, L3 Scholten, F., Preusker, F., Elgner, S., et al. 2019b, A\&A, 632, L5 Sugita, S., Honda, R., Morota, T., et al. 2019, Science, 364, 268 Suzuki, H., Yamada, M., Kouyama, T., et al. 2018, Icarus, 300, 341 Tatsumi, E., Kouyama, T., Suzuki, H., et al. 2019, Icarus, 325, 153 Watanabe, S., Tsuda, Y., Yoshikawa, M., et al. 2017, Space Sci. Rev., 208, 3 Watanabe, S., Hirabayashi, M., Hirata, N., et al. 2019, Science, 364, 268 Wewel, F. 1996, Int. Arch. Photogram. Remote Sens., 31, 936

Zhang, W., Giese, B., Oberst, J., \& Jaumann, R. 1996, Int. Arch. Photogram. Remote Sens., 31, 1004

1 http://europlanet.dlr.de/MASCOT 